\section{Erektile Dysfunktion: sexuelles Selbstvertrauen stärken}

— Für Männer, die unter erektiler Dysfunktion leiden, gibt es derzeit drei Therapiekonzepte mit PDE-5-Hemmern: die einmal tägliche Therapie mit Tadalafil $\left(\right.$ Cialis $\left.^{\circledR}\right) 5 \mathrm{mg}$, die bedarfsabhängige Einnahme von Tadalafil $10 \mathrm{mg}$ oder $20 \mathrm{mg}$ mit einem Wirkzeitfenster von bis zu 36 Stunden sowie kürzer wirksame PDE-5-Hem-

mer als Bedarfstherapie (beispielsweise Sildenafil, Wirkdauer 4-6 h). Welche dieser Therapien vermag, das sexuelle Selbstvertrauen und die Zufriedenheit der Betroffenen am besten zu steigern? Eine Untersuchung von Rubio-Aurioles et al., die kürzlich auf dem 25. Kongress der European Association of Urology (EAU) vorge-

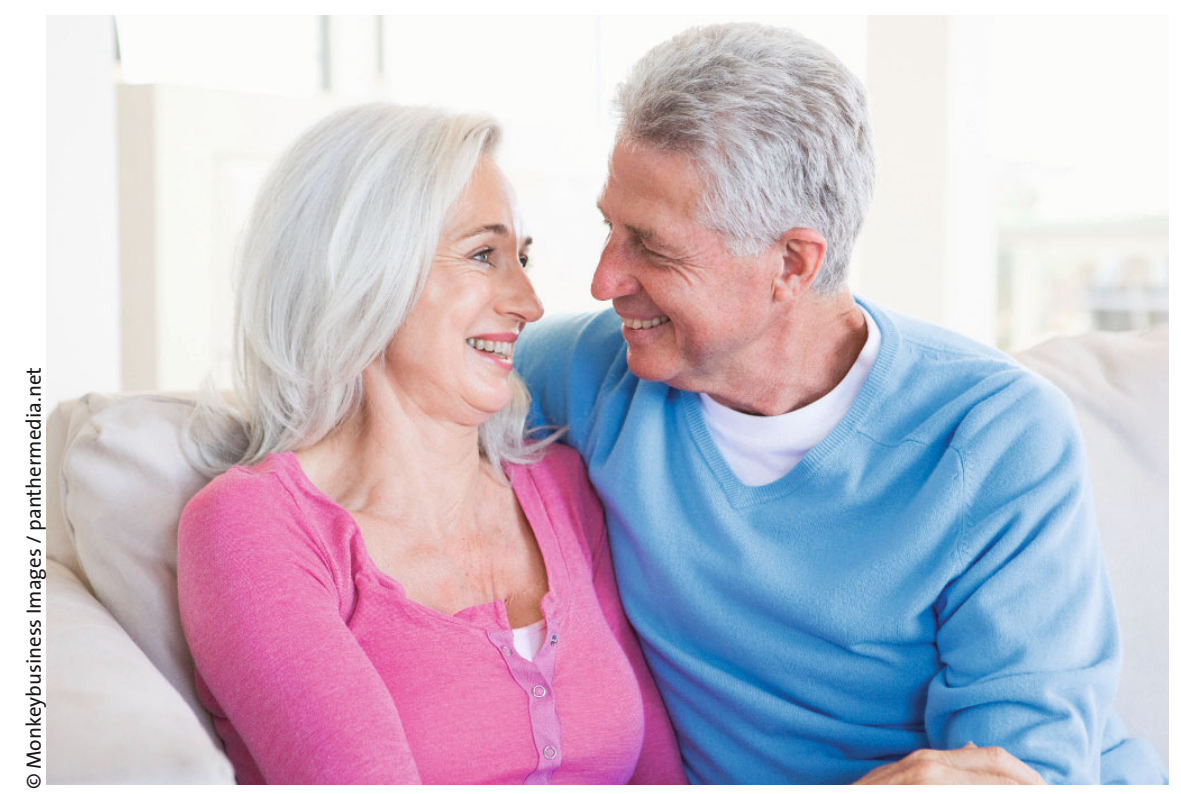

Durch eine Behandlung mit PDE-5-Hemmern steigt das sexuelle Selbstvertrauen. stellt wurde, hat sich mit dieser Frage beschäftigt. Die multizentrische, internationale Studie schloss insgesamt 378 Männer - Durchschnittsalter 56 Jahre - mit erektiler Dysfunktion ein. Im Verlauf der Studie erhielten die Patienten jeweils über acht Wochen $5 \mathrm{mg}$ Tadalafil einmal täglich, nach Bedarf $20 \mathrm{mg}$ Tadalafil sowie nach Bedarf 100 mg Sildenafil. Erfasst wurden anschließend das sexuelle Selbstvertrauen der Männer sowie die Wirksamkeit, Sicherheit und Verträglichkeit.

Unter allen Therapieregimes nahm das sexuelle Selbstvertrauen zu. Im Einzelnen zeigte sich, dass dies unter Therapie mit Tadalafil stärker ausfiel als unter Sildenafil. Zwischen den beiden Tadalafil-Optionen gab es keine signifikanten Unterschiede. Auch bei der Beurteilung der Spontanität beim Geschlechtsverkehr waren die Veränderungen unter den beiden Tadalafil-Therapien größer als unter Sildenafil. Außerdem nahm die Häufigkeit morgendlicher Erektionen bei der einmal täglichen Therapie mit Taldafil am stärksten zu: die Erektionen traten im Vergleich zur Bedarfstherapie an $6 \%$ mehr Tagen auf. Verglichen mit Sildenafil war dies sogar an $15 \%$ mehr Tagen der Fall.

Nach Informationen von

Lilly, Bad Homburg

\title{
Prostatakarzinom: Histrelin-Implantat steuert Testosteronspiegel
}

— Studien zeigen deutlich die Bedeutung eines gleichmäßig niedrigen Testosteronwertes während einer antiandrogenen Hormontherapie: Bei Patienten mit metastasiertem Prostatakarzinom existiert eine direkte Korrelation zwischen dem Serumtestosteronwert und dem spezifischen Sterberisiko. Das Histrelin-JahresImplantat Vantas ${ }^{\circledR}$ kann die Serumtestosteronwerte während der Behandlung dauerhaft unter $20 \mathrm{ng} / \mathrm{dl}$ senken und entspricht damit den Anforderungen an eine medikamentöse Androgensupppression. Darin waren sich die Experten auf dem Symposium des Unternehmens Orion Pharma im Rahmen des diesjährigen Kongresses der European Association of Urology (EAU) einig.
Zu den Vorteilen des Jahres-Implantats gegenüber anderen Vertretern der Substanzklassen zählt die gute Steuerbarkeit der Androgensuppression. Prof. Bertran Tombal, Brüssel, erklärt: „Die andiandrogene Wirkung von Histrelinacetat ist vergleichsweise rasch reversibel."

Vantas ${ }^{\circledR}$ enthält 50 mg Histrelin in einer Hydrogel-Depotformulierung. Durch seine Galenik erfolgt eine kontinuierliche Wirkstofffreisetzung von 50-60 $\mu \mathrm{g} /$ Tag über zwölf Monate. Dr. Neal D. Shore, Myrtle Beach, USA, stellte auf dem EAU-Kongress 2010 die Daten der Phase-III-Zulassungsstudie vor: „Das Jahres-Implantat erzielt mit im Median 11,8 ng/dl während des Therapieverlaufs im ersten Jahr Serumtestosteronwerte auf Kastrationsniveau."
Monatliche Messungen zeigten, dass die Testosteronwerte während des gesamten Therapieverlaufs stabil und niedrig blieben. Riskante Anstiege im Testosteronspiegel traten nicht auf, auch nicht beim Wechsel des Implantats nach 52 Wochen. Mit Vantas ${ }^{\circledR}$ kann demnach eine zuverlässige Testosteronsuppression erzielt werden, die Patienten mit fortgeschrittenem oder metastasiertem Prostatakarzinom eine weitere Option in der Langzeittherapie bietet.

Nach Informationen von

Orion Pharma, Hamburg 